\title{
Cicatrices de acné, un reto terapéutico
}

\author{
Acne scars, a therapeutic challenge
}

Nathalie Morales ${ }^{1}$, Ana María Aristizábal²

1. Médica, residente de tercer año de Dermatología, Universidad CES, Medellín, Colombia

2. Médica dermatóloga; docente, Universidad CES, Medellín, Colombia

\section{Resumen}

Las cicatrices por acné son una complicación frecuente y con grandes implicaciones sociales y psicológicas en el individuo, por lo cual la atención debe estar dirigida al manejo oportuno y agresivo de las formas graves de acné evitando así, en lo posible, su aparición. Sin embargo, algunos de estos pacientes van a presentar dicha complicación aun con una intervención temprana; esto se debe a una alteración en la cicatrización y a la gravedad y duración del fenómeno inflamatorio. Para su tratamiento se cuenta con diferentes alternativas químicas, físicas y quirúrgicas que podrían ser una buena opción para mejorar la apariencia de la piel.

Palabras Clave: acné, cicatrices, tratamiento.

\section{Summary}

Acne scars are a common complication with great social and psychological implications on the individual, so attention must be directed to the appropriate and aggressive management of severe forms of acne as soon as possible thus preventing its appearance. However, some of these patients will present this complication even with early intervention due to an impaired healing response and to the severity and duration of the inflammatory process. There are several chemical, physical and surgical treatment alternatives that could be a good choice for improving the appearance of the skin.

KEY WORDS: acne, scars, treatment.

\section{Correspondencia:}

Nathalie Morales

Email:

natymo_25@hotmail.com

Recibido: 5 de junio de 2013.

Aceptado: 15 de septiembre de 2013.

No se reportan conflictos de intereses.

\section{Introducción}

El acné es una enfermedad inflamatoria de la piel, que afecta a más del $80 \%$ de las personas entre los 11 y los 30 años y puede persistir en la edad adulta hasta en $5 \%$ de los casos ${ }^{1}$. Las formas inflamatorias de acné dan como resultado la formación de cicatrices permanentes, con sus respectivas implicaciones sociales y psicológicas en el individuo. Su prevalencia no ha sido bien estudiada, pero en algunos estudios se ha encontrado algún grado de cicatrización hasta en $95 \%$ de los pacientes con acné, con predominio en el área facial y, en menor proporción, en el tronco ${ }^{2}$.

Para entender la fisiopatología de las cicatrices es necesario conocer los factores implicados en el desarrollo de las lesiones de acné como la sobreproducción de sebo, la hiperqueratinización del folículo, la estimulación hormonal, la proliferación de Propionibacterium acnes y la respuesta inflamatoria ${ }^{3,4}$.

El proceso de cicatrización se lleva a cabo en tres fases. La primera es la fase inflamatoria durante la cual se blanquean las lesiones debido a la vasoconstricción 
para hacer hemostasia y, posteriormente, se produce eritema por la subsecuente vasodilatación, además de la hiperpigmentación secundaria a la activación de la melanogénesis, manifestación que es usualmente temporal pero que puede persistir de 8 a 12 meses $^{4,5}$. Esta fase es realmente importante puesto que en algunos estudios comparativos de acné con cicatrices y sin ellas, se ha encontrado una clara relación entre la duración prolongada y la mayor gravedad de la respuesta inflamatoria con la formación de cicatrices ${ }^{6}$.

La segunda es la fase de formación de tejido de granulación, en la cual hay regeneración del tejido dañado y activación de la angiogénesis, y posterior liberación de diferentes factores de crecimiento que estimulan la proliferación y diferenciación de los fibroblastos, con la consiguiente producción de colágeno; inicialmente, esta es rica en colágeno de tipo III (80 \%) y, en las cicatrices maduras, esta relación se invierte con una mayor proporción de colágeno de tipo I. Por último, se da una fase de remodelación de la matriz extracelular, en la que los fibroblastos y queratinocitos producen enzimas como las metaloproteinasas y sus inhibidores para reorganizar la arquitectura. Al generarse en esta fase un desequilibrio entre las metaloproteinasas y sus inhibidores, si la reacción es inadecuada con disminución del tejido colágeno, se forman cicatrices atróficas, o si, por el contrario, la reacción es exagerada con producción de abundante tejido fibrótico, se forman cicatrices hipertróficas ${ }^{4}$. También, se han identificado otros factores como la infección, el trauma repetido y la formación de quistes por largo tiempo que favorecen este proceso de formación de cicatrices ${ }^{5}$.

Las cicatrices de acné se clasifican en atróficas e hipertróficas. Las atróficas son las más comunes, se forman por pérdida del tejido colágeno y se localizan frecuentemente en el área facial. Estas cicatrices se subclasifican según su amplitud, profundidad y forma, en cicatrices en picahielo, rolling y boxcar.

Aquellas en picahielo, corresponden a 60 a $70 \%$ de las cicatrices, con una base estrecha de hasta $2 \mathrm{~mm}$, puntiforme pero profunda. Se describen en forma de "V" por su salida estrecha y un tracto epitelial que se extiende en profundidad en la dermis o tejido celular subcutáneo. Debido a estas características, los tratamientos superficiales no son de utilidad en este subtipo.

Las cicatrices en rolling corresponden a 15 a $35 \%$ de los casos. Son de base ancha, entre 4 y $5 \mathrm{~mm}$, y se deben a una alteración de las fibras de anclaje que conectan la dermis con la hipodermis, lo que les da un aspecto ondulado en forma de "M" o con arrugas sutiles en la superficie de la piel.

Las cicatrices en boxcar, corresponden a 20 a $30 \%$ de los casos. Se caracterizan por sus bordes verticales bien

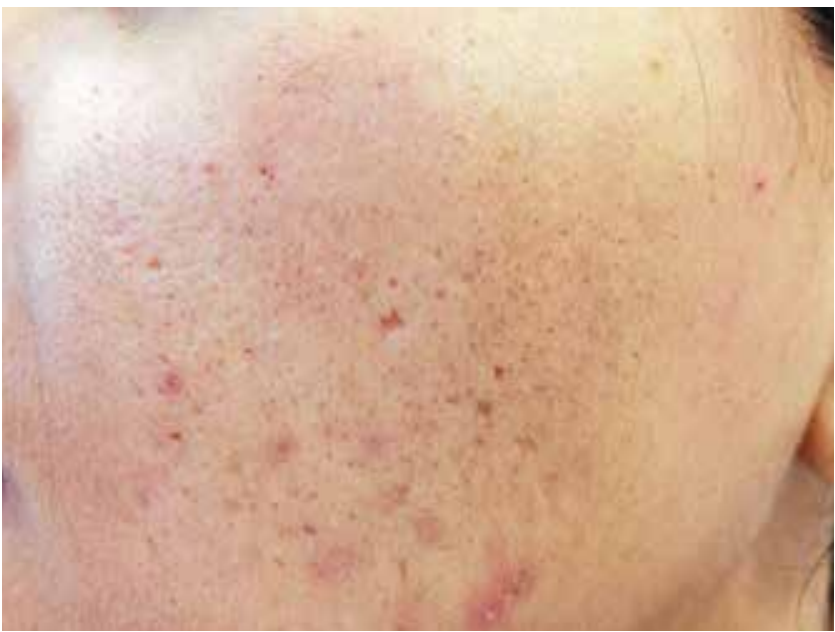

FIGURA 1. Cicatrices en boxcar y en picahielo.

delimitados, con una base más amplia que las cicatrices en picahielo, sin terminación cónica, lo que les confiere una apariencia ovalada o en "U", similar a la de las cicatrices por varicela. Se subdividen en superficiales $(<0,5$ $\mathrm{mm})$ y profundas $(>0,5 \mathrm{~mm})$ y tienen un diámetro entre 1,5 y $4 \mathrm{~mm}^{4-6}$ (FIGURA 1).

Las cicatrices hipertróficas y queloides, son otra variedad que, aunque no son específicas del acné, pueden presentarse como resultado de dicha enfermedad o ser secundarias a los tratamientos instaurados para otras cicatrices. Se localizan preferentemente en la cara anterior del tórax, la espalda, los hombros, el cuello y el ángulo de la mandíbula. En cuanto a sus diferencias clínicas, las cicatrices hipertróficas crecen en un periodo de tres meses después de la lesión inicial, son de textura blanda y superficie lisa; sus bordes limitan la lesión inicial sin sobrepasarla y tienden a involucionar con el paso de los años. Por otro lado, están los queloides, con un crecimiento lento en meses a años, superficie enrollada e indurada, con bordes que se extienden más allá de la lesión inicial y tendencia a persistir o aumentar de tamaño con el tiempo ${ }^{5,7}$.

Aparte de la clasificación de Jacob, et al., según el tipo de clínico, Goodman y Baron propusieron una clasificación en grados con una descripción cualitativa de la cicatriz así:

- Grado 1 o macular: eritema, con hiperpigmentación asociada o sin ella; el cambio es mayor en el color que en la textura de la piel.

- Grado 2 o leve: atrofia o hipertrofia muy leve; que requieren visualización a menos de $50 \mathrm{~cm}$ para ser visibles y pueden cubrirse con maquillaje. En este grado se encuentran las cicatrices en rolling.

- Grado 3 o moderado: atrofia o hipertrofia moderada; 
puede ser visible a más de $50 \mathrm{~cm}$ de distancia, es difícil de cubrir con maquillaje y puede aplanarse con el estiramiento manual de la piel. En esta clasificación se encuentran las cicatrices rolling y boxcar superficiales, y las hipertróficas o queloides papulares.

- Grado 4 o grave: atrofia o hipertrofia grave; es visible completamente a más de $50 \mathrm{~cm}$ de distancia, no se puede cubrir con maquillaje y no se aplanan con el estiramiento de la piel. A este grado corresponden las cicatrices en picahielo, las boxcar profundas y las hipertróficas o queloides de gran tamaño ${ }^{4,8}$.

El tratamiento de las cicatrices de acné está dirigido a mejorar la apariencia de la piel, teniendo en cuenta las características de las cicatrices (color, textura, profundidad, localización y número), los tratamientos previos, la presencia de lesiones inflamatorias de acné, el fototipo de piel y las expectativas del paciente. Además, hay que tener siempre en mente los efectos secundarios del tratamiento, como son el aumento de la cicatrización, la pigmentación posinflamatoria, las infecciones y la fibrosis. El tratamiento se puede dividir en dos grandes grupos: quirúrgico y no quirúrgico ${ }^{5,7,9}$.

\section{Métodos no quirúrgicos para cicatrices atróficas}

Se cuenta con diferentes modalidades de tratamientos, entre las cuales se encuentran la exfoliación cutánea (peeling), la microdermoabrasión y la dermoabrasión, los rellenos dérmicos o subcutáneos y el láser.

\section{Exfoliación cutánea (peeling)}

Este tipo de abrasión se produce con agentes exfoliantes que producen destrucción y promueven la regeneración de las diferentes capas de la piel. En numerosos estudios se trata sobre la utilidad de la exfoliación cutánea en el tratamiento de las cicatrices de acné ${ }^{4}$.

Uno de estos productos es el ácido tricloroacético, un agente cáustico que actúa causando precipitación de las proteínas, necrosis de coagulación en la epidermis y regeneración del colágeno en la dermis, lo que permite la remodelación y reorganización de la arquitectura, que pueden persistir varios meses.

La profundidad de su efecto en la piel depende de la concentración usada. Las concentraciones mayores de $35 \%$ se consideran una exfoliación media a profunda, con acción en la dermis papilar y reticular, que es lo que se desea alcanzar en cicatrices profundas como las en picahielo, pero con resultados aún impredecibles en cuanto a efectos secundarios.

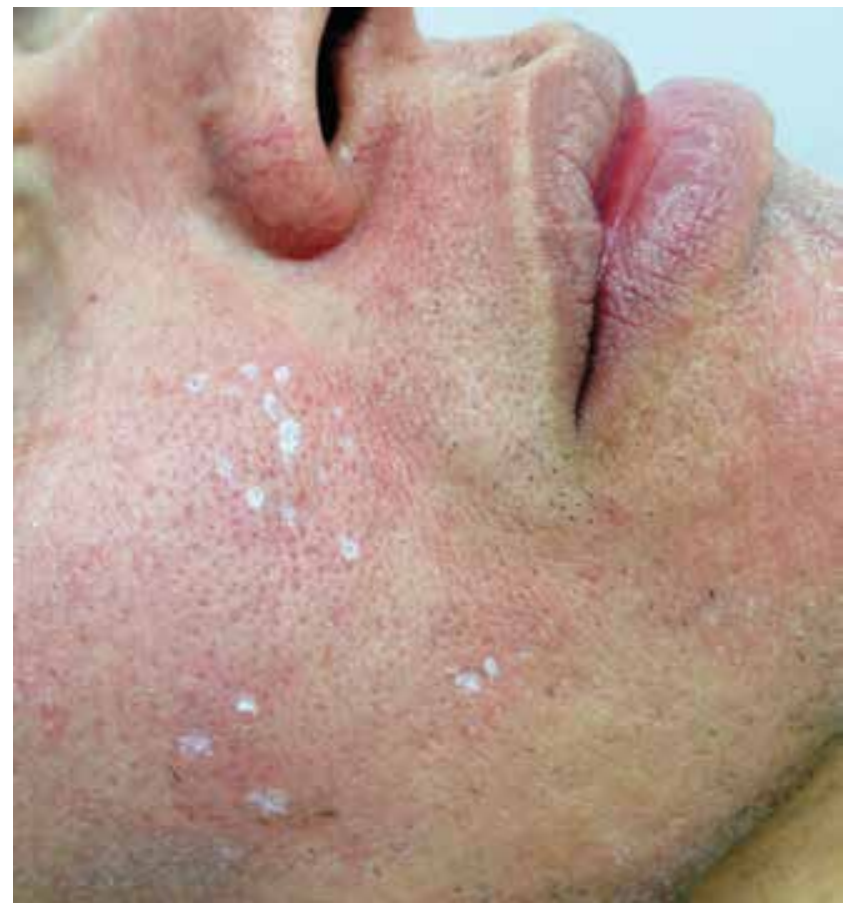

FIGURA 2. Reconstrucción clínica de cicatrices dérmicas (CROSS) mediante exfoliación cutánea con ácido tricloroacético al 35\%.

Hace varios años, Lee, et al., describieron por primera vez una técnica llamada reconstrucción clínica de cicatrices dérmicas (Clinical Reconstruction of Skin Scars, CROSS), en la cual se aplica de forma puntual en cada cicatriz ácido tricloroacético en concentraciones mayores o iguales a 90 \%, formando un área de escarcha sobre la cicatriz. Esta técnica se asocia a una curación más rápida y menor probabilidad de complicaciones. En revisiones más recientes se habla de concentraciones menores de $35 \%$ para evitar cambios en la pigmentación y aumento de las cicatrices; incluso, con la técnica CROSS usando concentraciones máximas de $50 \%$, se han obtenido resultados similares a los observados con mayores concentraciones. La aplicación debe hacerse cada cuatro semanas por tres sesiones, con énfasis en la fotoprotección y en el uso de emolientes en los siguientes siete días ${ }^{4,10,11}$ (FIGURA 2).

Hay otros agentes exfoliantes como el ácido salicílico, un beta-hidroxiácido, de elección en cicatrices de acné en piel de fototipo alto, por su menor probabilidad de hiperpigmentación postinflamatoria. Su efecto queratolítico se debe a la remoción de los lípidos intracelulares que se unen en forma covalente con las células epiteliales cornificadas. En cicatrices superficiales de acné, su uso a una concentración del $30 \%$ ha sido satisfactorio, con aplicaciones cada cuatro semanas por tres a cinco sesiones $^{12,13}$. 
El ácido glicólico es un alfa-hidroxiácido que aumenta el ácido hialurónico y la expresión de colágeno en la dermis, además de inducir epidermólisis y dispersión de la melanina, lo cual genera una acción queratolítica, antiinflamatoria y antioxidante. El mejor resultado en cicatrices de acné se obtuvo con una concentración de $70 \%$, con intervalos de dos semanas por cinco sesiones ${ }^{4,13,14}$.

El ácido pirúvico es un alfa-cetoácido con efecto queratolítico y sebostático que, además, genera producción de colágeno. Se usa en concentraciones de 40 a $70 \%$ en cicatrices de leve a moderada profundidad, pero tiene un uso limitado por la irritación secundaria en la piel y la mucosa respiratoria ${ }^{15}$.

Por último, la solución de Jessner, combinación de ácido salicílico al $14 \%$, ácido láctico al $14 \%$ y resorcinol al $14 \%$ en etanol, es de gran utilidad como tratamiento combinado para cicatrices superficiales ${ }^{13}$.

\section{Dermoabrasión y microdermoabrasión}

Son procedimientos utilizados para el rejuvenecimiento facial, con diferentes usos en la dermatología, entre ellos, las cicatrices por acné. La dermoabrasión es una técnica en la que se usa un dermoabrasivo con diferentes piezas de mano como fresas de diamante, cepillos de alambre, lijas o el moto-tool (motor o turbina quirúrgica) $\mathrm{Al}$ ejercer fricción sobre la piel, estos elementos generan erosión en la epidermis y en profundidad hasta la dermis papilar y reticular media, con el fin de estimular la reepitelialización secundaria y la remodelación del colágeno.

Este procedimiento está indicado para cicatrices superficiales rolling y boxcar y para mejorar la apariencia de las cicatrices más profundas, como las en picahielo. Como es una técnica abrasiva, debe practicarse bajo anestesia general, ya que puede ser muy dolorosa; además, deben tenerse en cuenta los efectos secundarios, como las infecciones, las cicatrices hipertróficas y las discromías ${ }^{16-18}$.

Por otro lado, la microdermoabrasión es un procedimiento que se basa en la utilización de un agente abrasivo, que usualmente es cristales de óxido de aluminio o puntas de diamante, y un componente de presión al vacío, lo que produce una exfoliación superficial en la epidermis y cambios en la dermis, como engrosamiento, proliferación de fibroblastos y depósito de colágeno. Los mejores resultados se han observado en los cambios de textura de la piel y de la pigmentación posinflamatoria $\mathrm{y}$, en menor proporción, en cicatrices superficiales en las cuales deben practicarse múltiples sesiones con intervalos mínimos de dos semanas y con una presión suficiente con la pieza de mano para generar rocío san- grante o punteado hemorrágico. Los efectos secundarios con esta técnica son mínimos, tales como el eritema y la descamación en los primeros días de practicado el procedimiento $^{18-20}$.

\section{Rellenos dérmicos o subcutáneos}

Los diversos materiales de relleno para el tratamiento de las cicatrices de acné, poseen diferentes características como longevidad (permanentes, semipermanentes o temporales), origen (bovino, humano o sintético) y funcionalidad ${ }^{9}$.

Los materiales ideales de relleno deben ser fisiológicos, fáciles de aplicar, y tener buena permanencia en la piel y mínimos efectos secundarios. Entre ellos se encuentran el ácido hialurónico, el colágeno bovino o humano, la grasa autóloga, ácido poli-L-láctico (Sculptra $\left.{ }^{\circledR}\right)$, el polimetil-metacrilato y las siliconas ${ }^{21,22}$.

El primer material aprobado por la Food and Drug Administration (FDA) para rellenos dérmicos fue el colágeno, usado para generar volumen y levantar la base de la cicatriz. Su tiempo de duración es corto, aproximadamente de tres a seis meses, por lo que requiere aplicaciones de mantenimiento. El ArteFill@ es un material de relleno compuesto de millones de microesferas sintéticas de poli-metil-metacrilato suspendidas en colágeno bovino. En la actualidad se conoce su utilidad en cicatrices profundas con buenos resultados y gran persistencia en el tiempo y, más recientemente, se confirmó su seguridad a pesar de contener material bovino que podría generar reacciones de hipersensibilidad ${ }^{21,22}$.

El ácido hialurónico es el principal polisacárido de la matriz extracelular, actúa como un sostén para el tejido colágeno y la elastina, y tiene una gran afinidad para unirse y atraer moléculas de agua; además, se ha visto que, tras su depósito en la dermis, tiene un efecto indirecto que es la activación de fibroblastos; por esto, es el más utilizado actualmente ${ }^{22,23}$.

Hay diferentes materiales de relleno a base de ácido hialurónico en el mercado, los cuales son de origen sintético y no requieren prueba previa de alergia. Estos productos varían según su dureza o maleabilidad, permanencia, capacidad de volumen y resistencia a la degradación enzimática. En general, pueden durar hasta nueve meses e incluso más tiempo según el tipo de ácido hialurónico; en cicatrices se ha reportado resultados de hasta un año de duración ${ }^{21-24}$.

El ácido poli-L-láctico, un material aprobado por la FDA para el tratamiento de la lipodistrofia producida por el VIH, se ha descrito como una opción terapéutica adecuada en cicatrices de difícil tratamiento, ya que tiene un efecto activador sobre los fibroblastos para la producción de colágeno, con una duración en el tejido 
de más o menos 18 a 24 meses; en algunos estudios preliminares ha mostrado resultados satisfactorios para esta finalidad, con mínimos efectos secundarios ${ }^{3,21,25,26}$.

La grasa autóloga es otra alternativa para el tratamiento de las cicatrices atróficas. Las limitaciones para su uso son la variabilidad en las tasas de duración en cada individuo, y que requiere liposucción de grasa del mismo paciente, una adecuada irrigación para sobrevivir en el tejido por largo tiempo y mayor tiempo en su aplicación en comparación con otros materiales de relleno ${ }^{22,27}$.

Uno de los métodos de relleno más usado en el pasado fue la aplicación de silicona líquida en la dermis profunda, que produce un efecto de volumen y fibroplasia del tejido a largo plazo, lo que permite encapsular el material de relleno e impedir su migración. Anteriormente, se hablaba de múltiples efectos secundarios, como granulomas de tipo parafinoma, reacciones inflamatorias y autoinmunitarias, e infecciones locales. En la actualidad, estos efectos son cada vez menos frecuentes, debido a la perfección en la técnica y la pureza de los productos. En el 2005 se publicó un estudio de Barnett y Barnett, en el que se demostró la eficacia y seguridad a largo plazo de las siliconas, en pacientes con cicatrices por acné. La silicona líquida se considera un método permanente y sin posibilidad de degradación, por lo que su aplicación debe hacerse de forma cautelosa para evitar resultados no deseados ${ }^{23,28,29}$.

\section{Láser}

Las cicatrices de acné pueden tratarse con láser ablativo o no ablativo, según la profundidad que se desea alcanzar y el tipo de cicatriz. Los láser ablativos tienen diferentes grados de afinidad por el agua, por lo cual su principal punto de acción está dirigido a la epidermis (compuesta hasta en el $90 \%$ por agua) y, además, genera un daño térmico residual en la dermis.

Entre los más usados están el láser $\mathrm{CO}_{2}$, con una menor selectividad por el agua pero con capacidad de generar desnaturalización del colágeno de tipo I y formación de nuevo colágeno, y de promover la reparación tisular. En varios estudios se ha demostrado mejoría de las cicatrices atróficas en 50 a $80 \%$ con el láser $\mathrm{CO}_{2}$. Los efectos secundarios son diversos e incluyen edema, formación de costras, quistes de millium, eritema persistente e hiperpigmentación hasta en $36 \%$ de los pacientes, principalmente aquellos con fototipos altos.

Por otro lado, está el laser erbium: yttrium-aluminumgarnet (Er: YAG) con una gran afinidad por el agua, el cual genera menor daño térmico en el tejido, por lo cual produce menos efectos secundarios con recuperación más rápida, pero menor efectividad que el láser $\mathrm{CO}_{2}{ }^{30-33}$.

Debido al tiempo requerido para la recuperación con los láser ablativos y las efectos secundarios, se introdujo el uso de láser no ablativos, como el Nd-YAG (neodymium: yttrium-aluminium-garnet) de $1.320 \mathrm{~nm}$ y 1.064 $\mathrm{nm}$, y el diodo láser de $1.450 \mathrm{~nm}$.

En diferentes estudios se ha demostrado la efectividad del láser Nd-YAG de $1.064 \mathrm{~nm}$ con ancho de pulso largo, y el diodo de $1.450 \mathrm{~nm}$ en el tratamiento de las cicatrices rolling y boxcar, en personas con piel de fototipos IV $\mathrm{y} \mathrm{V}$, pero pocos resultados en cicatrices en picahielo. Aunque tienen menos efectos secundarios, estos tipos de láser son menos efectivos que la ablación ${ }^{34-36}$.

Tratando de encontrar un punto medio entre efectividad y menos complicaciones y cuidados posteriores al procedimiento, se desarrolló la teoría de la fototermólisis fraccionada, la cual consiste en producir zonas microscópicas de daño térmico rodeadas por piel sana en forma de cuadrícula o patrón geométrico independiente del cromóforo. Esta técnica facilita la remodelación de la arquitectura a partir del tejido indemne, al actuar sobre la epidermis y la dermis, con menor daño térmico y recuperación más rápida. Existen diferentes tipos de láser fraccionado, entre ellos los ablativos como el Fraxel ${ }^{\circledR}$ y el Pixel®, y los no ablativos ${ }^{37,38}$.

\section{Métodos quirúrgicos en cicatrices atróficas}

\section{Subcisión}

Fue descrita por primera vez por Orentreich en 1995. Es la técnica quirúrgica preferida en cicatrices atróficas, en la cual se hace una incisión subcutánea para liberar las bridas o adherencias entre la dermis y el tejido celular subcutáneo, lo que permite la liberación funcional de la cicatriz y produce un trauma controlado que favorece la formación de nuevo colágeno durante el proceso de cicatrización de la herida.

Esta técnica se lleva a cabo con una aguja de calibre 18 a 20 o una aguja Nokor, la cual se inserta en la piel adyacente a la cicatriz de forma paralela a su superficie y con el bisel hacia arriba, inicialmente con movimientos hacia atrás y hacia adelante y luego en forma de abanico debajo de la cicatriz, esto con el fin de liberar las adherencias en el plano subcutáneo o dérmico. Al terminar, se ejerce compresión para evitar la formación de hematomas y se aconseja la aplicación de hielo local y antiinflamatorios durante cinco días.

Se requieren múltiples sesiones con intervalos de mínimo tres semanas y se logran tasas de mejoría de la apariencia de la piel de 40 a $50 \%$. No se recomienda esta técnica en la región preauricular, temporal o man- 

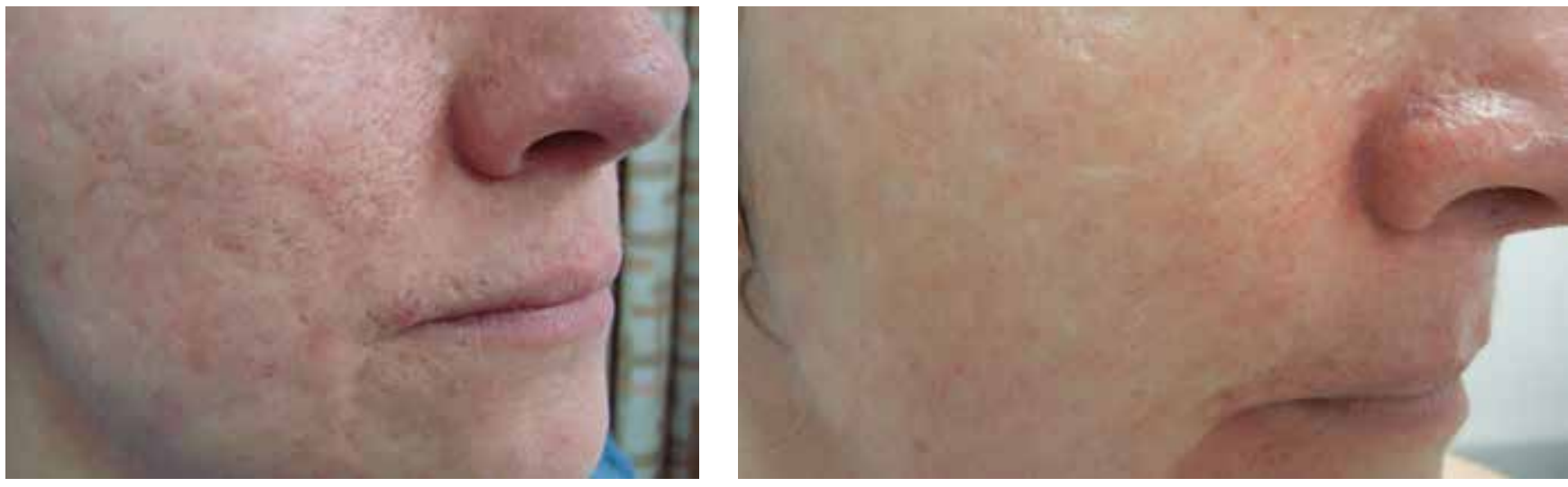

FigURA 3. (A) Cicatrices en rolling y boxcar. (B) Resultado después de dos sesiones de subcisión y resección con sacabocados.

dibular, con el fin de evitar lesiones en el nervio facial y los grandes vasos ${ }^{1,21,39,40}$. En el 2010 se describió una técnica de subcisión y succión con el microdermabrador, encontrándose con esta combinación un aumento de la mejoría entre 60 y $90 \%$, sin efectos secundarios importantes ${ }^{41}$ (FIGURA 3).

\section{Técnicas con sacabocados}

Existen tres modalidades de tratamiento con indicación específica para cicatrices en picahielo y Boxcar profundas.

1. Escisión en sacabocados. Esta técnica consiste en practicar una escisión con sacabocados de 1,5, $305 \mathrm{~mm}$, el cual debe abarcar toda la cicatriz con sus bordes incluidos y llegar en profundidad hasta el tejido celular subcutáneo para asegurar la remoción del tejido cicatricial. Luego se sutura con Prolene ${ }^{\circledR}$ 5-0 o 6-0, con puntos separados en suficiente cantidad para permitir un adecuado afrontamiento de la herida quirúrgica.

Si se requiere una escisión de mayor tamaño, es preferible recurrir a la escisión elíptica, con el fin de evitar defectos en el cierre como las "orejas de perro". En general, se recomienda dejar espacios entre cada cicatriz de mínimo 4 a $5 \mathrm{~mm}$, para evitar retracciones, y un intervalo de cuatro semanas entre los procedimientos, para permitir una adecuada cicatrización. Los puntos deben retirarse a los siete días 3 ,42,43,51.

2. Incisión y elevación con sacabocados. Esta técnica que combina la escisión con sacabocados y los microinjertos es de gran utilidad en cicatrices deprimidas superficiales y profundas. Se deja piel flotante solo en los bordes laterales de la cicatriz y se lleva el tejido a una altura un poco mayor que la de la piel adyacente. Esto permite que, al retraerse el tejido durante el proceso de cicatrización, deje una apariencia más elevada de la piel afectada. Al final debe fijarse la piel flotante al tejido circundante con sutura o con una gota de pegamento de cianoacrilato ${ }^{42-44}$.

3. Microinjertos. Los microinjertos de piel requieren de una cuidadosa técnica para que el injerto sobreviva en la zona receptora. Se usan específicamente en cicatrices en picahielo, debido a las dificultades con los otros tratamientos por la profundidad de la lesión. Consiste en la resección con un sacabocados de las cicatrices seleccionadas hasta el tejido celular subcutáneo (área receptora). El tejido resecado se reemplaza con un injerto de piel de espesor total tomado de la región retroauricular. Se debe ocluir para proteger el injerto y garantizar su permanencia, y se esperan siete días, tiempo en el cual ya se ha adherido el injerto al tejido receptor ${ }^{45-47}$.

\section{Microagujas}

Esta técnica consiste en practicar punciones repetidas sobre la piel afectada para promover la eliminación y producción de nuevo colágeno. Se requiere un rodillo estéril compuesto de una serie de agujas afiladas, las cuales se aplican en la piel con cierto grado de presión y con movimientos en varias direcciones en las áreas afectadas, penetrando hasta $1,502 \mathrm{~mm}$ en la dermis.

Es una técnica fácil, con mínimos efectos secundarios, como sangrado transitorio y equimosis local, con resultados satisfactorios desde la sexta semana hasta los tres meses después del tratamiento, y una mejoría adicional en la textura de la piel en los 12 meses siguientes.

Está contraindicada en pacientes con anticoagulación 
o infecciones activas en piel, en quienes se haya aplicado materiales de relleno en los últimos seis meses y si hay antecedentes de cicatrices hipertróficas o queloides ${ }^{4,48-51}$.

\section{Tratamiento combinado}

En muchos estudios se ha demostrado una mayor efectividad del tratamiento combinado en las cicatrices de acné, como: los rellenos dérmicos y el láser fraccionado; la exfoliación cutánea y las microagujas o la dermoabrasión; y la exfoliación cutánea seguida de subcisión y láser fraccionado ${ }^{52-55}$. También se han descrito técnicas quirúrgicas combinadas entre ellas y asociadas con láser fraccionado ${ }^{1}$.

\section{¿Qué hay de nuevo?}

En los últimos años se ha hablado de la utilidad del plasma rico en plaquetas como una alternativa terapéutica en la curación de heridas, debido a sus propiedades moduladoras y estimuladoras de la proliferación y diferenciación de las células de origen mesenquimatoso, entre ellas los fibroblastos y las células endoteliales, lo que favorece la regeneración tisular.

El plasma rico en plaquetas es una fracción de plasma obtenido de la sangre del mismo paciente, con una gran concentración de plaquetas, superior a la normal. Estas plaquetas contienen un gran número de factores de crecimiento y citocinas, los cuales son secretados activamente por los gránulos plaquetarios, además de proteínas involucradas en la adhesión celular, como la fibronectina y fibrina; proporcionan un medio de soporte para la diferenciación y proliferación de los fibroblastos, y la reparación y regeneración de la matriz extracelular. La técnica más frecuentemente descrita para su obtención es la centrifugación, pero en nuestro medio se hace también por secuestro gravitacional de las plaquetas, evitando la centrifugación excesiva y mejorando la calidad de la muestra.

Una vez obtenido el plasma rico en plaquetas, este puede permanecer estable durante ocho horas en condiciones de anticoagulación. En el momento de su aplicación debe adicionarse una sustancia activadora, como el cloruro de calcio y la trombina, para permitir la liberación de los gránulos plaquetarios y, por ende, su acción. Una vez agregado el activador, debe procederse a la aplicación del plasma antes de 10 minutos para evitar la retracción del coágulo, y el secuestro de las proteínas $\mathrm{y}$ factores derivados de las plaquetas.

El plasma rico en plaquetas se ha estudiado para cirugía maxilofacial, ortopedia y, en dermatología, en el tratamiento contra el envejecimiento y en la reepitelia- lización de úlceras venosas, pero son pocos los estudios sobre la reparación de cicatrices de acnéé,57.

En un estudio de Lee, et al., sobre el tratamiento combinado con láser $\mathrm{CO}_{2}$ y aplicación intradérmica de plasma rico en plaquetas o solución salina, se demostró una recuperación más rápida y mayor mejoría de las cicatrices con dicho plasma. Esto nos lleva a pensar en la necesidad de futuros estudios controlados que demuestren su efectividad como tratamiento único en las cicatrices de acnés8.

\section{Tratamiento de las cicatrices hipertróficas y queloides}

En cuanto a las cicatrices hipertróficas y queloides, la mejor opción continúa siendo el tratamiento combinado con la infiltración intralesional de esteroides, crioterapia e, incluso, en algunos casos, resección quirúrgica.

Hay otros tratamientos alternativos, como la silicona en parches por dos a tres meses, el láser, la radioterapia y la aplicación intralesional de medicamentos como el 5-fluoracilo, la bleomicina y el interferón alfa, con los cuales se han obtenido grados variables de mejoría en diferentes estudios ${ }^{1,4,59}$.

En conclusión, las cicatrices por acné son una complicación con gran impacto psicológico y de difícil de manejo, debido a que no hay un tratamiento totalmente efectivo y con resultados rápidos. Por esto, se han usado tratamientos combinados con las diferentes opciones terapéuticas que mejoran los resultados a largo plazo; además, se han desarrollado nuevas tecnologías con buenos resultados, como le fototermólisis selectiva y, más recientemente, el plasma rico en plaquetas. Este último requiere de más estudios en el tratamiento de las cicatrices del acné para poder hacer recomendaciones basadas en la evidencia científica. Debido a la dificultad en su manejo, se recomienda el tratamiento oportuno y adecuado del acné para prevenir su aparición.

\section{Referencias}

1. Jacob CI, Dover JS, Kaminer MS. Acne scarring: A classification system and review of treatment options. J Am Acad Dermatol. 2001;45:109-17.

2. Layton AM, Henderson CA, Cunliffe WJ. A clinical evaluation of acne scarring and its incidence. Clin Exp Dermatol. 1994;19:303-8.

3. Levy LL, Zeichner JA. Management of acne scarring, part II: A comparative review of non-laser-based, minimally invasive approaches. Am J Clin Dermatol. 2012;13:331-40.

4. Fabbrocini G, Annunziata MC, D'Arco V, De Vita V, Lodi G, Mauriello MC, et al. Acne scars: Pathogenesis, classification and treatment. Dermatol Res Pract. 2010;2010:1-13. 
5. Tsao SS, Dover JS, Arndt KA, Kaminer MS. Scar management: Keloid, hypertrophic, atrophic, and acne scars. Semin Cutan Med Surg. 2002;21:46-75.

6. Holland DB, Jeremy AH, Roberts SG, Seukeran DC, Layton AM, Cunliffe WJ. Inflammation in acne scarring: A comparison of the responses in lesions from patients prone and not prone to scar. $\mathrm{Br}$ J Dermatol. 2004;150:72-81.

7. Basta-Juzbašić A. Current therapeutic approach to acne scars. Acta Dermatovenerol Croat. 2010;18:171-5.

8. Goodman GJ, Baron JA. The management of postacne scarring. Dermatol Surg. 2007;33:1175-88.

9. Grupo Colombiano de Estudio en Acné. Guías colombianas para el manejo del acné: una revisión basada en la evidencia. Rev Asoc Colomb Dermatol. 2011;19:129-58.

10. Slavin JW. Trichloroacetic acid peels. Aesthetic Surgery Journal. 2004;24:469-70.

11. Bhardwaj D, Khunger N. An assessment of the efficacy and safety of CROSS technique with $100 \%$ TCA in the management of ice pick acne scars. J Cutan Aesthet Surg. 2010;3:93-6.

12. Grimes PE. The safety and efficacy of salicylic acid chemical peels in darker racial-ethnic groups. Dermatol Surg 1999;25:18-22.

13. Landau M. Chemical peels. Clin Dermatol. 2008;26:200-8.

14. Erbağci Z, Akçali C. Biweekly serial glycolic acid peels Vs. long-term daily use of topical low-strength glycolic acid in the treatment of atrophic acne scars. Int J Dermatol. 2000;39:789-94.

15. Berardesca E, Cameli N, Primavera G, Carrera M. Clinical and instrumental evaluation of skin improvement after treatment with a new 50\% pyruvic acid peel. Dermatol Surg. 2006;32:526-31.

16. Kim EK, Hovsepian RV, Mathew P, Paul MD. Dermabrasion. Clin Plast Surg. 2011;38:391-5.

17. Campbell RM, Harmon CB. Dermabrasion in our practice. J Drugs Dermatol. 2008;7:124-8.

18. Alkhawam L, Alam M. Dermabrasion and microdermabrasion. Facial Plast Surg. 2009;25:301-10.

19. Tsai RY, Wang CN, Chan HL. Aluminum oxide crystal microdermabrasion. A new technique for treating facial scarring. Dermatol Surg. 1995;21:539-42.

20. Karimipour DJ, Karimipour G, Orringer JS. Microdermabrasion: An evidence-based review. Plast Reconstr Surg. 2010;125:372-7.

21. Rivera AE. Acne scarring: A review and current treatment modalities. J Am Acad Dermatol. 2008;59:659-76.

22. Glogau RG. Fillers: From the past to the future. Semin Cutan Med Surg. 2012;31:78-87.

23. Sánchez I, Candelas D, Ruiz R. Materiales de relleno: tipos, indicaciones y complicaciones. Actas Dermosifiliogr. 2010;101:381-93.

24. Bisaccia E, Saap L, Kadry R, Scarborough D. Non-invasive procedures in cosmetic dermatology. Skin Aging. 2007;15:38-40.

25. Sadove R. Injectable poly-L-lactic acid: A novel sculpting agent for the treatment of dermal fat atrophy after severe acne. Aesthetic Plast Surg. 2009;33:113-6.

26. Sadick NS, Palmisano L. Case study involving use of injectable poly-L-lactic acid (PLLA) for acne scars. Journal of Dermatological Treatment. 2009;20:302-7.
27. Pinski KS, Roenigk HH Jr. Autologous fat transplantation. Longterm follow-up. J Dermatol Surg Oncol. 1992;18:179-84.

28. Barnett JG, Barnett CR. Treatment of acne scars with liquid silicone injections: 30-year perspective. Dermatol Surg. 2005;31:1542-9.

29. Prather CL, Jones DH. Liquid injectable silicone for soft tissue augmentation. Dermatol Ther. 2006;19:159-68.

30. Carniol PJ, Meshkov L, Grunebaum LD. Laser treatment of facial scars. Curr Opin Otolaryngol Head Neck Surg. 2011;19:283-8.

31. Alexiades-Armenakas MR, Dover JS, Arndt KA. The spectrum of laser skin resurfacing: Nonablative, fractional, and ablative laser resurfacing. J Am Acad Dermatol. 2008;58:719-37.

32. Walia S, Alster TS. Prolonged clinical and histologic effects from $\mathrm{CO}_{2}$ laser resurfacing of atrophic acne scars. Dermatol Surg. 1999;25:926-30.

33. Tanzi EL, Alster TS. Single-pass carbon dioxide versus multiplepass Er:YAG laser skin resurfacing: A comparison of postoperative wound healing and side-effect rates. Dermatol Surg. 2003;29:80-4

34. Chua SH, Ang P, Khoo LS, Goh CL. Nonablative 1450-nm diode laser in the treatment of facial atrophic acne scars in type IV to V Asian skin: A prospective clinical study. Dermatol Surg. 2004;30:1287-91.

35. Lee DH, Choi YS, Min SU, Yoon MY, Suh DH Comparison of a 585-nm pulsed dye laser and a 1064-nm Nd:YAG laser for the treatment of acne scars: A randomized split-face clinical study. J Am Acad Dermatol. 2008;60:801-7.

36. Min SU, Choi YS, Lee DH, Yoon MY, Suh DH. Comparison of a long pulse Nd:YAG laser and a combined 585/1,064-nm laser for the treatment of acne scars: A randomized split-face clinical study. Dermatol Surg 2009;35:1720-7

37. Hasegawa T, Matsukura T, Mizuno Y, Suga Y, Ogawa H, Ikeda S. Clinical trial of a laser device called fractional photothermolysis system for acne scars. J Dermatol. 2006;33:623-7.

38. Manstein D, Herron GS, Sink RK, Tanner H, Anderson RR. Fractional photothermolysis: A new concept for cutaneous remodeling using microscopic patterns of thermal injury. Lasers Surg Med. 2004;34:426-38.

39. Chandrashekar B, Nandini A. Acne scar subcision. J Cutan Aesthet Surg. 2010;3:125-6

40. Al-Dhalimi MA, Arnoos AA. Subcision for treatment of rolling acne scars in Iraqi patients: A clinical study. J Cosmet Dermatol. 2012;11:144-50.

41. Aalami S, Balighi K, Lajevardi V, Akbari E. Subcision-suction method: A new successful combination therapy in treatment of atrophic acne scars and other depressed scars. J Eur Acad Dermatol Venereol. 2011;25:92-9.

42. Khunger N. Standard guidelines of care for acne surgery. Indian J Dermatol Venereol Leprol. 2008;74:28-36.

43. AlGhamdi KM, AlEnazi MM. Versatile punch surgery. J Cutan Med Surg. 2011;15:87-96.

44. Khunger N; IADVL Task Force. Standard guidelines of care for acne surgery. Indian J Dermatol Venereol Leprol. 2008;74:28-36.

45. Johnson WC.Treatment of pitted scars. Punch transplant technique. J Dermatol Surg Oncol. 1986;12:260.

46. de Almeida AR, de Oliveira NI, Kadunc BV, de Almeida Prado Sampaio S. Surgical pearl: Lubricating jelly as a dressing in punch grafts for pitted acne scars. J Am Acad Dermatol. 1998;38:613-5. 
47. Goodman GJ. Management of post-acne scarring. What are the options for treatment? Am J Clin Dermatol. 2000;1:3-17.

48. Fabbrocini G, Fardella N, Monfrecola A, Proietti I, Innocenzi D. Acne scarring treatment using skin needling. Clin Exp Dermatol. 2009;34:874-9.

49. Majid I. Microneedling therapy in atrophic facial scars: An objective assessment. J Cutan Aesthet Surg. 2009;2:26-30.

50. Doddaballapur S. Microneedling with dermaroller. J Cutan Aesthet Surg. 2009 1;2:110-1.

51. Fife D. Practical evaluation and management of atrophic acne scars: Tips for the general dermatologist. J Clin Aesthet Dermatol. 2011;4:50-7.

52. Ayhan S, Baran CN, Yavuzer R, Latifoglu O, Cenetoglu S, Baran NK. Combined chemical peeling and dermabrasion for deep acne and post-traumatic scars as well as aging face. Plast Reconstr Surg. 1998;102:1238-46.

53. O'Daniel TG. Multimodal management of atrophic acne scarring in the aging face. Aesthetic Plast Surg. 2011;35:1143-50.
54. Sharad J. Combination of microneedling and glycolic acid peels for the treatment of acne scars in dark skin. J Cosmet Dermatol. 2011;10:317-23.

55. Kang WH, Kim YJ, Pyo WS, Park SJ, Kim JH. Atrophic acne scar treatment using triple combination therapy: Dot peeling, subcision and fractional laser. J Cosmet Laser Ther. 2009;11:212-5.

56. Rodríguez J, Palomar MA, Torres J. Plasma rico en plaquetas: fundamentos biológicos y aplicaciones en cirugía maxilofacial y estética facial. Rev Esp Cir Oral Maxilofac. 2012;34:8-17.

57. Kim DH, Kim JY, Seo SH, Ahn HH, Kye YC, Choi JE. Recalcitrant cutaneous ulcer of comorbid patient treated with platelet rich plasma: A case report. J Korean Med Sci. 2012;27:1604-6.

58. Lee JW, Kim BJ, Kim MN, Mun SK. The efficacy of autologous platelet rich plasma combined with ablative carbon dioxide fractional resurfacing for acne scars: A simultaneous split-face trial. Dermatol Surg. 2011;37:931-8.

59. Juckett G, Hartman-Adams H. Management of keloids and hypertrophic scars. Am Fam Physician. 2009;80:253-60. 